\title{
Clinical Factors for Prognosis and Treatment Guidance of Spinal Cord Astrocytoma
}

\author{
Toshitaka Seki ${ }^{1}$, Kazutoshi Hida ${ }^{2}$, Shunsuke Yano ${ }^{2}$, Takeshi Aoyama ${ }^{3}$, \\ Izumi Koyanagi ${ }^{4}$, Toru Sasamori ${ }^{1}$, Shuji Hamauch ${ }^{1}$, Kiyohiro Houkin ${ }^{1}$ \\ ${ }^{I}$ Department of Neurosurgery, Hokkaido University Graduate School of Medicine, Sapporo, Japan \\ ${ }^{2}$ Department of Neurosurgery, Sapporo Azabu Neurosurgical Hospital, Sapporo, Japan \\ ${ }^{3}$ Department of Orthopedic Surgery, Teine Keijinkai Hospital, Sapporo, Japan \\ ${ }^{4}$ Department of Neurosurgery, Hokkaido Neurosurgical Memorial Hospital, Sapporo, Japan
}

\begin{abstract}
Study Design: Retrospective study.
Purpose: To obtain information useful in establishing treatment guidelines by evaluating baseline clinical features and treatment outcomes of patients with spinal cord astrocytoma (SCA).

Overview of Literature: The optimal management of SCA remains controversial, and there are no standard guidelines.

Methods: The study included 20 patients with low-grade and 13 with high-grade SCA surgically treated between 1989 and 2014. Patients were classified according to the extent of surgical resection. Survival was assessed using Kaplan-Meier plots and compared between groups by log-rank tests. Neurological status was defined by the modified McCormick scale and compared between groups by Mann-Whitney U tests.

Results: Surgical resection was performed for 19 of 20 low-grade (95\%) and 10 of 13 high-grade (76.9\%) SCA patients. Only nine patients (27.3\%) underwent gross total resection, all of whom had low-grade SCA. Of all patients, 51.5\% showed deteriorated neurological status compared to preoperative baseline. Median overall survival was significantly longer for low-grade SCA than that for high-grade SCA (91 months, $78 \%$ at 5 years vs. 15 months, $31 \%$ at 5 years; $p=0.007$ ). Low-grade SCA patients benefited from more aggressive resection, whereas high-grade SCA patients did not. Multivariate analysis revealed histology status (hazard ratio [HR], 0.30; $95 \%$ confidence interval [Cl], 0.09-0.98; $p<0.05$ ) and postoperative neurological status (HR, $0.12 ; \mathrm{Cl}, 0.02-0.95 ; p<0.05$ ) as independent predictors of longer overall survival. Adjuvant radiotherapy had no significant impact on survival rate. However, a trend for increased survival was observed with radiation cordotomy (RCT) in high-grade SCA patients.

Conclusions: Aggressive resection for low-grade and RCT for high-grade SCA may prolong survival. Preservation of neurological status is an important treatment goal. Given the low incidence of SCA, establishing strong collaborative, interdisciplinary, and multiinstitutional study groups is necessary to define optimal treatments.
\end{abstract}

Keywords: Astrocytoma; Intramedullary tumor; Outcome; Radiotherapy; Surgery

\section{Introduction}

Intramedullary spinal cord tumors are rare neoplasms that account for only $2 \%-4 \%$ of all central nervous system tumors $[1,2]$. Among them, spinal cord astrocytomas (SCAs) represent only $6 \%-8 \%$ of spinal cord tumors [3-5].

\footnotetext{
Received Oct 26, 2015; Revised Nov 17, 2015; Accepted Dec 6, 2015

Corresponding author: Toshitaka Seki

Department of Neurosurgery, Hokkaido University Graduate School of Medicine,

N-15, W-7, Kita-ku Sapporo, Hokkaido 0608638, Japan

Tel: +81-11-706-5987, Fax: +81-11-708-7737, E-mail: toseki1@hotmail.com
} 
Of all SCAs, $7 \%-30 \%$ are malignant $[3,6,7]$. However, optimal management guidelines for this neoplasm have yet to be defined. The survival benefits of radical or gross total resection (GTR) of low-grade astrocytomas are inconsistent in the literature [4,5,8-10]. For high-grade SCAs, many authors have proposed limited resection because of their infiltrating nature, lack of distinct cleavage planes, and overall poor prognosis regardless of therapeutic intervention [10-14]. The aim of this study was to obtain prognostic information useful for establishing management guidelines by examining the clinical records of SCA patients treated at Hokkaido University Hospital.

\section{Materials and Methods}

The clinical outcomes of SCA patients treated from 1989 to 2014 at Hokkaido University Hospital were reviewed. Data were collected from clinical, radiographic, operative, and pathological reports. All study participants provided informed consent, and the study design was approved by our institutional ethics review board.

The degrees of resection were classified as GTR, subtotal resection (STR), partial resection (PR), or biopsy. GTR was defined as the complete removal of the lesion intraoperatively and/or the postoperative absence of the lesion on T1-weighted gadolinium-enhanced magnetic resonance imaging. STR was defined as the removal of $\geq 90 \%$, PR as resection of $<90 \%$, and biopsy as resection of a minimal amount of tumor tissue for the purpose of diagnosis. We applied a modified McCormick classification (Table 1) [15] to assess neurological function before surgery and at the date of death or final follow-up.

The patients were divided into two groups based on the pathological tumor grade: a low-grade SCA group (World Health Organization [WHO] grades I and II) and a highgrade SCA group (grades III and IV).

\section{Statistical analyses}

In the low-grade SCA group, we compared the change in neurological function after surgery using the MannWhitney U test between the GTR or STR group, and the PR or biopsy group. In the high-grade SCA group, the change in neurological function after surgery was compared between the GTR, STR, or PR group and the biopsy group. Overall survival (OS) and 5-year survival were estimated using the Kaplan-Meier method [16] and compared among groups by the log-rank test. OS was defined as the time from diagnosis to death or the censored date of the last follow-up. Univariate analyses were performed to determine the effect of various patient, tumor, and treatment variables on OS. Multivariate Cox proportional hazard models were used to calculate hazard ratios (HRs) and $95 \%$ confidence intervals (CIs) to investigate independent predictors of OS rate. All variables were assessed in a stepwise multivariate model that required a $p<0.1$

Table 1. Modified McCormick classification

\begin{tabular}{|c|c|}
\hline Grade & Definition \\
\hline I & $\begin{array}{l}\text { - Neurologically normal } \\
\text { - Gait normal } \\
\text { - Normal professional activity }\end{array}$ \\
\hline $\mathrm{lb}$ & $\begin{array}{l}\text { - Tired after walking several kilometers } \\
\text { - Running is impossible, or moderate sensorimotor deficit significantly affect the involved limb } \\
\text { - Moderate discomfort in professional activity }\end{array}$ \\
\hline$\|$ & $\begin{array}{l}\text { - Presence of sensorimotor deficit affecting function of involved limb } \\
\text { - Mild to moderate gait difficulty } \\
\text { - Severe pain or dysesthetic syndrome impairs } \\
\text { - Quality of life } \\
\text { - Independent function and ambulation maintained }\end{array}$ \\
\hline III & $\begin{array}{l}\text { - More severe neurological deficit } \\
\text { - Requires cane and/or brace for ambulation or maintains significant bilateral upper-extremity impairment } \\
\text { - May or may not function independently }\end{array}$ \\
\hline IV & $\begin{array}{l}\text { - Severe neurological deficit } \\
\text { - Requires wheelchair or cane and/or brace with bilateral upper-extremity impairment } \\
\text { - Usually not independent }\end{array}$ \\
\hline
\end{tabular}


for variable entry and retention. $p<0.05$ was considered statistically significant. All analyses were performed using Ekuseru-Toukei 2015 (Social Survey Research Information Co. Ltd., Tokyo, Japan).

\section{Results}

\section{Patient cohort}

Thirty-three consecutive SCA patients were included in the analysis. Five (15.2\%) had pilocytic astrocytoma (WHO grade I), 15 (45.5\%) WHO grade II astrocytoma, nine (27.3\%) anaplastic astrocytoma (WHO grade III), and four (12.1\%) glioblastoma (GBM; WHO grade IV). The median age of low-grade SCA patients was 36 years (mean, 38.9 years) and 55\% were female. The median age of high-grade SCA was 52 years (mean, 42.6 years) and $69.2 \%$ were male. Patients with high-grade SCA were older, but not significantly so $(p=0.61)$. The median duration of follow-up was 91 months (range, 2-261 months) for low-grade SCA and 15 months (range, 2-174 months) for high-grade SCA.

\section{Tumor characteristics}

Low-grade SCAs most frequently occurred in the cervicothoracic spinal cord (40\%), followed by the cervical and thoracic (25\%), and thoracolumbar (10\%) regions, whereas high-grade SCAs occurred most frequently in the thoracic spinal cord (38.5\%), followed by thoracolumbar (30.8\%), cervical $(23.1 \%)$, and cervicothoracic $(7.7 \%)$ regions (Fig. 1).

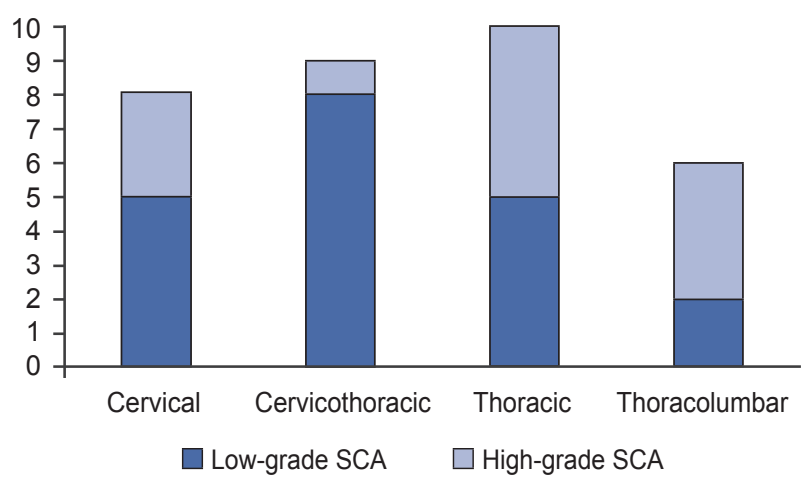

Fig. 1. Tumor characteristics of all 33 patients with spinal cord astrocytoma (SCA).

\section{Treatment}

Surgical resection was the most common treatment in the entire cohort (87.9\%), with the remaining patients receiving only biopsy for diagnosis. In patients with low-grade SCA, GTR was performed most often (45\%), followed by PR (30\%), STR (20\%), and biopsy (5\%). In contrast, no high-grade patient received GTR; the most common procedures were PR (69.2\%), followed by biopsy (23.1\%), and STR (7.7\%).

Postoperatively, the majority of patients with SCA received radiotherapy or chemotherapy (63.6\%). Of those with low-grade SCA, 15\% received chemotherapy and $25 \%$ radiotherapy. One low-grade SCA patient exhibited malignant transformation and underwent radiation cordotomy (RCT). All high-grade SCA patients received radiation therapy, nine following chemotherapy, and four received RCT.

\section{Preoperative and postoperative neurological status}

Preoperative neurological status according to the modified McCormick scale was grade I in four patients, grade Ib in seven, grade II in 14, grade III in three, and grade IV in five patients. Modified McCormick scale at final followup was grade I in three patients, grade Ib in three, grade II in eight, grade III in six, and grade IV in 13 patients. Eleven patients with low-grade SCA (55\%) and six patients with high-grade SCA (46.2\%) showed deterioration of neurological function at final follow-up (Tables 2, 3). In low-grade patients, neurological function after surgery was more likely to be improved or unchanged in patients receiving GTR or STR compared to those receiving PR or biopsy $(p=0.054)$. In the high-grade group, however, neurological deteriorated was significant after GTR, STR, or PR compared to biopsy $(p=0.041)$.

\section{Survival outcomes}

Median survival for all SCA patients was 53 months (62\% at 5 year) (Fig. 2A). Median survival was significantly longer for low-grade SCA patients than for high-grade SCA patients (91 months, $78 \%$ at 5 years vs. 15 months, $31 \%$ at 5 years; $p=0.007$ ) (Fig. $2 \mathrm{~B}$ ). Low-grade SCA patients who underwent GTR or STR had a significantly higher survival rate than those who underwent $\mathrm{PR}$ or biopsy ( 177 vs. 80 months, $91 \%$ vs. $54 \%$ at 5 years; $p=0.016$ ) 
Table 2. Summary of patients of low-grade spinal cord astrocytoma at the final follow-up

\begin{tabular}{|c|c|c|c|c|c|c|}
\hline \multirow{2}{*}{ Preoperative MMS } & \multicolumn{6}{|c|}{ MMS at the final follow-up } \\
\hline & I & $\mathrm{lb}$ & II & III & IV & Total \\
\hline I & 3 & & 1 & & & 4 \\
\hline lb & & 2 & 2 & & 1 & 5 \\
\hline$\|$ & & & 2 & 3 & 3 & 8 \\
\hline III & & & & 1 & 1 & 2 \\
\hline IV & & & & & 1 & 1 \\
\hline Total & 3 & 2 & 5 & 4 & 6 & 20 \\
\hline
\end{tabular}

MMS, modified McCormick scale.

Table 3. Summary of patients of high-grade spinal cord astrocytoma at the final follow-up

\begin{tabular}{|c|c|c|c|c|c|c|}
\hline \multirow{2}{*}{ Preoperative MMS } & \multicolumn{6}{|c|}{ MMS at the final follow-up } \\
\hline & I & $\mathrm{lb}$ & II & III & IV & Total \\
\hline I & & & & & & 0 \\
\hline $\mathrm{lb}$ & & 1 & 1 & & & 2 \\
\hline$\|$ & & & 2 & 2 & 2 & 6 \\
\hline III & & & & & 1 & 1 \\
\hline IV & & & & & 4 & 4 \\
\hline Total & 0 & 1 & 3 & 2 & 7 & 13 \\
\hline
\end{tabular}

MMS, modified McCormick scale.
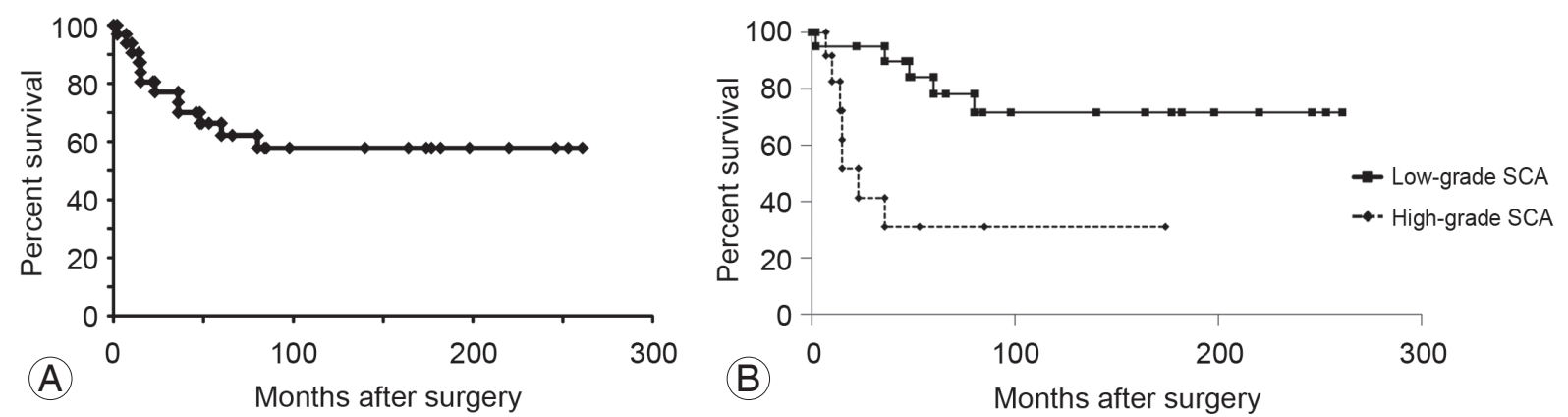

Fig. 2. Survival rates in the study. (A) Survival rates for all 33 patients with SCA. (B) Survival was significantly longer for low-grade SCA compared to high-grade SCA ( $p=0.007)$. SCA, spinal cord astrocytoma.

(Fig. 3A). For high-grade SCA patients, however, there was no significant difference in median survival time between the resection group (receiving GTR, STR, or PR) and the biopsy group (14.5 months vs. 23 months, $26 \%$ vs. $50 \%$ at 5 years; $p=0.44$ ) (Fig. $3 \mathrm{~B}$ ). Therefore, surgical resection did not impact median survival of patients with high-grade SCA. Median survival of SCA patients receiving RCT was higher than that of patients not receiving
RCT (38 vs. 10 months, $50 \%$ vs. $19 \%$ at 5 years), although the difference did not reach significance $(p=0.30)$ (Fig. 3C) as only 4 patients received RCT.

Univariate analysis of variables affecting OS (Table 4) revealed that low-grade histology was significantly related to longer survival (HR, 0.22 ; 95\% CI, $0.07-0.70 ; p=0.01$ ) while a poorer final follow-up modified McCormick scale grade was associated with significantly shorter survival 

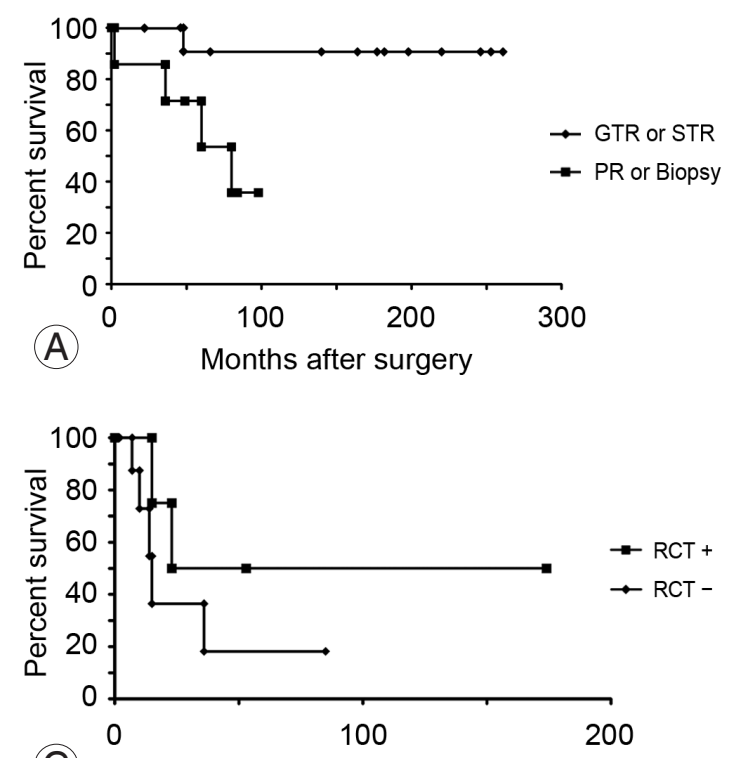

Months after surgery

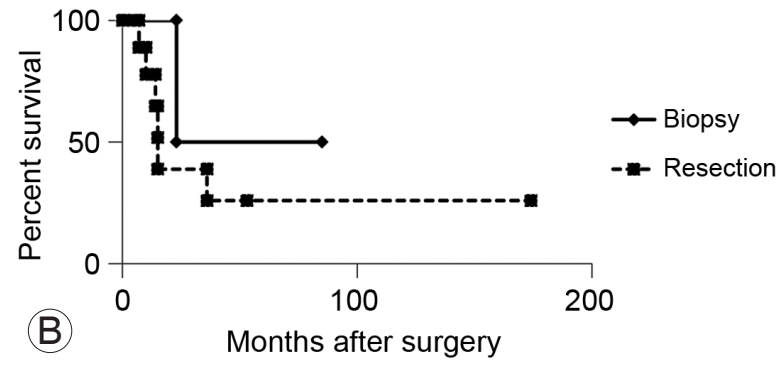

Fig. 3. Survival rates for patients with SCA. (A) Survival rates for patients with low-grade SCA after extensive or minimal surgical resection. Patients who underwent GTR or STR showed a significantly higher survival rate than those who underwent PR or biopsy $(p=0.016)$. (B) Survival rates for patients with high-grade SCA after surgical resection or biopsy. There was no significant difference in survival between the resection group (GTR, STR, or PR) and biopsy group $(p=0.44)$. (C) Survival rates for high-malignancy patients who underwent RCT. Although there was no significant difference between RCT and non-RCT groups in this small sample $(p=0.30)$, patients treated with RCT showed substantially longer median and higher 5-year survival. SCA, spinal cord astrocytoma; GTR, gross total resection; STR, subtotal resection; PR, partial resection; RCT, radiation cordotomy.

Table 4. Univariate analysis of various patient, tumor, treatment, and outcome characteristics

\begin{tabular}{lcc} 
Characteristic & HR $(95 \% \mathrm{CI})$ & $p$-value \\
\hline Age ( $\geq 39$ yr vs. <39 yr) & $1.62(0.51-5.15)$ & 0.41 \\
\hline Sex (male vs. female) & $2.11(0.63-7.06)$ & 0.23 \\
\hline Lesion: incl. or not incl. cervical spinal cord & $1.05(0.34-3.26)$ & 0.94 \\
\hline Tumor characteristics: low-grade SCA vs. high-grade SCA & $0.22(0.07-0.70)$ & $0.01^{\text {a) }}$ \\
\hline Preoperative MMS: I, II vs. III, IV & $0.55(0.16-1.83)$ & 0.33 \\
\hline Final follow-up MMS: I, Ib, II vs. III, IV & $0.09(0.01-0.74)$ & $0.02^{\text {a) }}$ \\
\hline Resection rate: resection vs. biopsy & $0.39(0.10-1.47)$ & 0.16 \\
\hline Radiation therapy: yes vs. no & $2.46(0.73-8.22)$ & 0.14 \\
\hline Chemotherapy: yes vs. no & $2.37(0.76-7.41)$ & 0.14 \\
\hline
\end{tabular}

HR, hazard ratio; 95\% Cl, 95\% confidence interval; incl., including; SCA, spinal cord astrocytoma; MMS, modified McCormick scale. a) Significant difference.

Table 5. Multivariate Cox proportional hazard models for patients with SCA

\begin{tabular}{lcc} 
Characteristics & HR $(95 \% \mathrm{CI})$ & $p$-value \\
\hline Tumor characteristics: low-grade SCA vs. high-grade SCA & $0.30(0.09-0.98)$ & $0.0469^{a)}$ \\
\hline Final follow-up MMS: I, Ib, II vs. III, IV & $0.12(0.02-0.95)$ & $0.0443^{\text {a) }}$ \\
\hline
\end{tabular}

SCA, spinal cord astrocytoma; HR, hazard ratio; 95\% Cl, 95\% confidence interval; MMS, modified McCormick scale.

a) Significant difference.

(HR, 0.09; 95\% CI, 0.01-0.74; $p=0.002$ ). However, tumor resection rate was not significantly associated with OS improvement in SCA patients.

Multivariate stepwise analysis revealed that tumor his- tology (HR, 0.30; 95\% CI, 0.09-0.98; $p<0.05)$ and final follow-up modified McCormick scale grade (HR, 0.12; 95\% CI, 0.02-0.95; $p<0.05)$ were independent predictors of OS in SCA patients (Table 5). 


\section{Discussion}

The best management strategy for patients with SCA remains controversial, and there are currently no universally accepted treatment guidelines. As described in previous studies, the most significant predictor of SCA survival is pathological grade $[4,5,12]$. Similarly, in the present study, patients with low-grade SCA had a significantly better prognosis than those with high-grade SCA and appeared to benefit more, both in terms of survival and neurological outcome, from aggressive surgical resection.

The survival benefits of surgical resection are inconsistent across studies [4,17-22]. Some studies recommended aggressive resection [19,21-23], while others concluded that biopsy alone yielded better prognosis $[4,5,8,24]$. McGirt et al. [21] reported that radical resection was associated with a trend for longer survival of patients with anaplastic SCA but not for those with GBM multiforme. Furthermore, patients with anaplastic SCA who underwent radical resection had a higher OS rate than those who underwent subtotal resection. On the other hand, Minehan et al. [20] concluded that the extent of tumor resection did not impact OS of SCA patients; and in fact, there was a trend for poorer OS in patients who underwent more aggressive resection compared to those who underwent biopsy alone. The impact of resection may depend strongly on baseline tumor grade. In our cohort, patients with low-grade SCA who underwent GTR or STR showed significantly longer median survival than those who underwent PR or biopsy. In contrast, median survival was longer after biopsy than tumor resection in high-grade SCA patients, although the difference did not reach significance. A similar impact of tumor grade was observed for neurological function. There was a trend for patients with low-grade SCA to benefit neurologically from more extensive resection (GTA or STR) compared to patients receiving PR or biopsy. On the other hand, in patients with high-grade SCA, the resection (GTR, STR, or PR) to avoid neurological deterioration and decrease in survival rates may not be appropriate, because the OS rate after these procedures was poorer compared with biopsy alone.

In the present series, four high-malignancy SCA patients received RCT, including one patient with low-grade astrocytoma showing malignant transformation. Although there was no significant difference in OS between patients receiving RCT and those not $(p=0.30)$, the median survival of patients who underwent RCT was almost 5-fold longer and 5-year survival more than double. Thus, RCT may be justified in some cases of highly malignant tumors, at least for tumors located below the thoracic level [22,25].

High tumor grade has consistently been shown to decrease OS of patients with SCA, including in this study, with tumor grade being a significant variable in multivariate analysis (HR, 0.30; 95\% CI, 0.09-0.98; $p<0.05$ ) $[6,17,22,24]$. Furthermore, our study revealed that postoperative neurological status was the most significant factor predicting better prognosis (HR, 0.12; 95\% CI, 0.02-0.95; $p<0.05)$. In our cohort, $51.5 \%$ showed neurological deterioration compared to preoperative baseline, consistent with previous studies reporting a postoperative neurological decline in $21 \%-64 \%$ of patients $[17,21,22,24,26]$. Therefore, preventing neurological deterioration after treatment may improve SCA survival rate. In previous studies, tumor dissemination was also deemed to be a significant factor predicitive of shorter survival [10,14,21,24], but this was not considered in the current study.

There are two limitations to this study. First, it is retrospective in nature so we can make no conclusions on causality. Prospective studies are required to determine whether treatments such as radiotherapy or chemotherapy are effective for SCA. Another limitation is the relatively small sample size due to the rarity of SCA. Multicenter studies, including prospective trials, are clearly needed.

\section{Conclusions}

SCA is an uncommon and successful treatment remains difficult. For low-grade SCA, patients who undergo GTR or STR obtained better OS. On the other hand, treatments for patients with high-grade SCA, including surgery, radiation therapy, or chemotherapy, were unsatisfactory. Multivariate analysis for patients with SCA revealed highgrade histology and neurological status in final follow-up to be significant predictors of poor survival.

Prospective studies are required to define prognostic factors and establish the optimal treatment for SCA. However, the incidence of these lesions is low; hence, strong collaborative, interdisciplinary, and multi-institutional study groups are necessary.

\section{Conflict of Interest}

No potential conflict of interest relevant to this article was 
reported.

\section{References}

1. Stein BM. Surgery of intramedullary spinal cord tumors. Clin Neurosurg 1979;26:529-42.

2. Chamberlain MC, Tredway TL. Adult primary intradural spinal cord tumors: a review. Curr Neurol Neurosci Rep 2011;11:320-8.

3. Epstein FJ, Farmer JP, Freed D. Adult intramedullary astrocytomas of the spinal cord. J Neurosurg 1992;77: 355-9.

4. Minehan KJ, Shaw EG, Scheithauer BW, Davis DL, Onofrio BM. Spinal cord astrocytoma: pathological and treatment considerations. J Neurosurg 1995;83: 590-5.

5. Innocenzi G, Salvati M, Cervoni L, Delfini R, Cantore G. Prognostic factors in intramedullary astrocytomas. Clin Neurol Neurosurg 1997;99:1-5.

6. Bouffet E, Pierre-Kahn A, Marchal JC, et al. Prognostic factors in pediatric spinal cord astrocytoma. Cancer 1998;83:2391-9.

7. Hardison HH, Packer RJ, Rorke LB, Schut L, Sutton LN, Bruce DA. Outcome of children with primary intramedullary spinal cord tumors. Childs Nerv Syst 1987;3:89-92.

8. Sandler HM, Papadopoulos SM, Thornton AF Jr, Ross DA. Spinal cord astrocytomas: results of therapy. Neurosurgery 1992;30:490-3.

9. Samii M, Klekamp J. Surgical results of 100 intramedullary tumors in relation to accompanying syringomyelia. Neurosurgery 1994;35:865-73.

10. Cohen AR, Wisoff JH, Allen JC, Epstein F. Malignant astrocytomas of the spinal cord. J Neurosurg 1989;70: 50-4.

11. Cristante L, Herrmann HD. Surgical management of intramedullary spinal cord tumors: functional outcome and sources of morbidity. Neurosurgery 1994;35:69-74.

12. Constantini S, Miller DC, Allen JC, Rorke LB, Freed D, Epstein FJ. Radical excision of intramedullary spinal cord tumors: surgical morbidity and longterm follow-up evaluation in 164 children and young adults. J Neurosurg 2000;93:183-93.

13. Jallo GI, Freed D, Epstein F. Intramedullary spinal cord tumors in children. Childs Nerv Syst 2003;19:
641-9.

14. Santi M, Mena H, Wong K, Koeller K, Olsen C, Rushing EJ. Spinal cord malignant astrocytomas: clinicopathologic features in 36 cases. Cancer 2003;98:55461.

15. McCormick PC, Torres R, Post KD, Stein BM. Intramedullary ependymoma of the spinal cord. J Neurosurg 1990;72:523-32.

16. Kaplan EL, Meier P. Nonparametric estimation from incomplete observations. J Am Stat Assoc 1958;53: 457-81.

17. Kim MS, Chung CK, Choe G, Kim IH, Kim HJ. Intramedullary spinal cord astrocytoma in adults: postoperative outcome. J Neurooncol 2001;52:85-94.

18. Benes V 3rd, Barsa P, Benes V Jr, Suchomel P. Prognostic factors in intramedullary astrocytomas: a literature review. Eur Spine J 2009;18:1397-422.

19. Adams H, Avendano J, Raza SM, Gokaslan ZL, Jallo GI, Quinones-Hinojosa A. Prognostic factors and survival in primary malignant astrocytomas of the spinal cord: a population-based analysis from 1973 to 2007. Spine (Phila Pa 1976) 2012;37:E727-35.

20. Minehan KJ, Brown PD, Scheithauer BW, Krauss WE, Wright MP. Prognosis and treatment of spinal cord astrocytoma. Int J Radiat Oncol Biol Phys 2009;73: 727-33.

21. McGirt MJ, Goldstein IM, Chaichana KL, Tobias ME, Kothbauer KF, Jallo GI. Extent of surgical resection of malignant astrocytomas of the spinal cord: outcome analysis of 35 patients. Neurosurgery 2008;63:55-60.

22. Nakamura M, Chiba K, Ishii K, et al. Surgical outcomes of spinal cord astrocytomas. Spinal Cord 2006; 44:740-5.

23. Cooper PR, Epstein F. Radical resection of intramedullary spinal cord tumors in adults: recent experience in 29 patients. J Neurosurg 1985;63:492-9.

24. Babu R, Karikari IO, Owens TR, Bagley CA. Spinal cord astrocytomas: a modern 20-year experience at a single institution. Spine (Phila Pa 1976) 2014;39:53340.

25. Seki T, Hida K, Yano S, Aoyama T, Koyanagi I, Houkin K. Surgical outcomes of high-grade spinal cord gliomas. Asian Spine J 2015;9:935-41.

26. Lee HK, Chang EL, Fuller GN, et al. The prognostic value of neurologic function in astrocytic spinal cord glioma. Neuro Oncol 2003;5:208-13. 